Short Note

\title{
Microwave Synthesis of (4-hydroxy Phenyl) 3-oxo butanoate
}

\author{
Mohammed Ramdani *, Nour-Eddine Benchat, Abderrahmane Anaflous, Fouad El Kalai \\ Applied Chemistry and Environement Laboratory, Faculty of Sciences, Univerity Mohamed 1st, \\ Oujda, Morooco
}

* Author to whom correspondence should be addressed. Fax : +212 365006 03;

E-Mail: moharamdani2000@yahoo.fr

Received: 2 May 2007; in revised form: 20 November 2007 / Accepted: 15 January 2008 / Published:

25 March 2008<smiles>CCOC(=O)CC(C)=O</smiles>

1

2

3

The product 3 was previously prepared [1] using $\mathrm{C}_{6} \mathrm{H}_{6}$ in the presence of pyridine. A mixture of hydroquinone 1 ( $0.66 \mathrm{~g}, 6 \mathrm{mmol})$, ethyl acetoacetate $2(0.78 \mathrm{~g}, 6 \mathrm{mmol})$ and monmorillonite K10 (0.43 $\mathrm{g}, 30 \%$ by weight of the total reactants) $[2,3]$ was placed in a pyrex tube which was then introduced into a Maxidigest MX 350 Prolabo microwave monomode reactor fitted with a rotational system [4]. An approximate final temperature $\left(120^{\circ} \mathrm{C}\right)$ was measured by introducing a digital thermometer at the end of the irradiation time (20 min on $180 \mathrm{~W}$ as irradiation power). The mixture was cooled to ambient temperature. After elution with ethyl acetate $(30 \mathrm{~mL})$ and subsequent filtration through florisil, the organic product was purified by chromatography on silicagel (dichloromethane : ethyl acetate, $90: 10$ ), yield : $65 \%$ of 3 white solid.

Melting point: $96-98^{\circ} \mathrm{C}$.

${ }^{1} \mathrm{H}$ NMR $\delta\left(\mathrm{CDCl}_{3}, 200 \mathrm{MHz}\right)$ : 7-6.7 (2d, 4H, Ph); 5,65 (s, OH) ; $3.7\left(\mathrm{~s}, \mathrm{CH}_{2}\right) ; 2.38\left(\mathrm{~s}, \mathrm{CH}_{3}\right)$.

${ }^{13} \mathrm{C}$ NMR $\delta\left(\mathrm{CDCl}_{3}, 100 \mathrm{MHz}\right): 30\left(\mathrm{CH}_{3}\right) ; 50\left(\mathrm{CH}_{2}\right) ; 116$ and $123\left(\mathrm{C}=\mathrm{C}\right.$ arom.); $155\left(\mathrm{CO}_{2}\right) ; 202(\mathrm{C}=\mathrm{O}$ acyl).

MS (IC-NH3, m / z): 212(M+ 18$) / 100 \%$.

\section{References}

1. Rall, K.B.; Perekalin, V.V. Zhurnal Obshchei Khimii 1955, 25, 259.

2. Li, T.S.; Zhang, Z.H.; Yang, F.; Fu, C.G. J. Chem. Research (S) 1998, 38-39.

3. Singh, J.; Kaur, J.; Nayyar, S.; Kad, G.L. J. Chem. Research (S) 1998, 280-281.

4. (a) Kappe, C.O.; Dallinger, D. Nature Reviews Drug Discovery 2006, 5, 51; (b) De la Hoz, A.; Diaz-Ortiz, A.; Moreno, A. Chem. Soc. Rev., 2005, 34, 164.

(C) 2008 by MDPI (http://www.mdpi.org/). Reproduction is permitted for noncommercial purposes. 